\title{
artigo
}

Oliveira, V.B.; Aguiar, R.S.

Conhecimento da política de saúde do homem e a relação com a atenção à saúde

\section{Conhecimento da política de saúde do homem e a relação com a atenção à saúde}

\author{
Knowledge of man's health policy and relationsgip to health care
}

Conocimiento de la política de salud del hombre y relación com la atendión de salud

\begin{abstract}
RESUMO
Objetivo: Analisar o conhecimento de enfermeiros e gestores da atenção primária à saúde sobre a Política Nacional de Atenção Integral a Saúde do Homem, bem como as suas repercussões na atenção à saúde. Método: Trata-se de uma revisão integrativa realizada nas bases de dados SciELO, LILACS e BDENF. Para a definição da pergunta norteadora utilizou-se a estratégia PICO e quanto ao processo de seleção dos estudos, utilizou-se o fluxograma PRISMA. Dessa forma, selecionaram-se 10 artigos para análise por meio da síntese narrativa. Resultados: Revela-se fragilidades no conhecimento dos profissionais e gestores acerca da política, influenciando negativamente na atenção integral do homem nos serviços de saúde. Conclusão: Os profissionais de saúde possuem pouco conhecimento acerca da política; e os gestores da atenção primária à saúde não possuem conhecimento suficiente para a implantação da mesma de modo que não estimulam os profissionais a se capacitarem para melhorar 0 atendimento dos homens.
\end{abstract}

DESCRITORES: Saúde do Homem; Atenção Primária à Saúde; Políticas de Saúde.

\section{ABSTRACT}

Objective: To analyze the knowledge of nurses and managers of primary health care about the National Policy for Integral Attention to Men's Health, as well as its repercussions on health care. Method: This is an integrative review carried out in the SciELO, LILACS and BDENF databases. For the definition of the guiding question, the PICO strategy was used and for the study selection process, the PRISMA flowchart was used. Thus, 10 articles were selected for analysis through the narrative synthesis. Results: It reveals weaknesses in the knowledge of professionals and managers about the policy, negatively influencing the integral attention of men in health services. Conclusion: Health professionals have little knowledge about the policy; and managers of primary health care do not have enough knowledge to implement it, so they do not encourage professionals to be trained to improve the care of men.

DESCRIPTORS: Men's Health; Primary Health Care; Health Policy.

\section{RESUMEN}

Objetivo: analizar el conocimiento de las enfermeras y gerentes de atención primaria de salud sobre la Política Nacional de Atención Integral a la Salud de los Hombres, así como sus repercusiones en la atención médica. Método: Esta es una revisión integradora realizada en las bases de datos SciELO, LILACS y BDENF. Para la definición de la pregunta guía, se utilizó la estrategia PICO y para el proceso de selección del estudio, se utilizó el diagrama de flujo PRISMA. Así, se seleccionaron 10 artículos para su análisis a través de la síntesis narrativa. Resultados: revela debilidades en el conocimiento de los profesionales y gerentes sobre la política, influyendo negativamente en la atención integral de los hombres en los servicios de salud. Conclusión: los profesionales de la salud tienen poco conocimiento sobre la política; y los gerentes de atención primaria de salud no tienen suficiente conocimiento para implementarla, por lo que no alientan a los profesionales a capacitarse para mejorar la atención de los hombres. DESCRIPTORES: Salud del Hombre; Atención Primaria de Salud; Política de Salud.

RECEBIDO EM: 22/06/2020 APROVADO EM: 08/07/2020

\section{Vanessa Braga Oliveira}

Enfermeira. Universidade Paulista (UNIP), Campus Brasília, Distrito Federal, Brasil.

ORCID: 0000-0002-7401-4216 


\section{Ricardo Saraiva Aguiar}

Professor Assistente. Curso de Graduação em Enfermagem, Universidade Paulista (UNIP), Campus Brasília, Distrito Federal, Brasil. ORCID: 0000-0003-0335-2194

\section{INTRODUÇÃO}

0 Ministério da Saúde lançou em 2009 a Política Nacional de Atenção Integral a Saúde do Homem (PNAISH) com a intenção de reduzir a morbimortalidade e ampliar o acesso dos homens aos serviços da atenção primária à saúde (APS), levando em consideração as atitudes e os comportamentos assumidos por estes nas relações sociais ${ }^{1}$.

O objetivo da PNAISH é promover a procura e ampliar o acesso da população masculina aos serviços de saúdel, sendo a primeira política de saúde da América Latina direcionada ao gênero masculino ${ }^{2}$, pois estes apresentam maior dificuldade em adotar comportamentos saudáveis e de procurarem o serviço de saúde, tendo uma maior vulnerabilidade e altas taxas de mortalidade.

Além disso, adentram o sistema de saúde normalmente pela atenção hospitalar de média e alta complexidade ${ }^{3}$, o que produz custos excedentes em internações hospitalares. Estas atitudes têm gerado como consequência o agravo das morbidades que poderiam ser evitadas caso realizassem com regularidade as medidas de prevenção primária ${ }^{3}$.

É comum entre a população masculina a ausência de cuidados voltados a sua saúde, muitas vezes, devido ao fator cultural. Assim, é preciso desenvolver ações para mobilizá-los para os serviços de saúde, principalmente na APS, sendo necessário que os profissionais estejam capacitados e consigam desenvolver ações para receberem esse público de forma acolhedora e consolidando o vínculo dos homens com as equipes de saúde, estabelecendo assim um cuidado concretizado ${ }^{1}$.

A APS deve ser a porta de entrada dos usuários no sistema de serviços de saúde de forma a satisfazer as principais necessidades por meio de um conjunto de intervenções individuais e coletivas que envolvem a promoção, a prevenção, o diagnóstico, o tratamento e a reabilitação do usuário ${ }^{5-6}$.
A APS deve ser a

porta de entrada

\section{dos usuários no}

sistema de serviços

de saúde de forma a

satisfazer as principais

necessidades por

meio de um conjunto

de intervenções

individuais e coletivas

que envolvem

a promoção, a

prevenção, 0

diagnóstico, o

tratamento e a

reabilitação do

usuário.
Nesse contexto, o presente estudo tem como objetivo analisar o conhecimento dos enfermeiros e dos gestores da APS sobre a PNAISH, bem como as suas repercussões na atenção à saúde. Sob essa perspectiva, destaca-se a questão que norteou a presente proposta investigativa: qual o conhecimento de enfermeiros e gestores da APS acerca da PNAISH e as suas repercussões na atenção à saúde?

\section{MÉTODO}

Trata-se de uma revisão integrativa da literatura, uma vez que contribui no processamento sistemático e analítico dos resultados, pois é característica da busca de informações sobre um assunto ou tema que resuma a situação da ciência sobre um problema de pesquisa, visando a clareza do determinado tema ${ }^{7}$.

Elaborou-se, na primeira fase, a pergunta norteadora de pesquisa. Para a construção dessa, utilizou-se da estratégia PICO: $\mathrm{P}$ - população e problema; I - intervenção; C - comparação; e O - outcome (termo em inglês que significa desfecho) 8 . Assim, considerou-se P: enfermeiros e gestores da APS; I: PNAISH; C: qualquer comparação entre o conhecimento da PNAISH; O: atenção à saúde do homem. Nesta direção, a pergunta construída foi: qual o conhecimento de enfermeiros e gestores da APS acerca da PNAISH e as suas repercussões na atenção à saúde?

Construiu-se para a segunda fase, uma estratégia de busca por dois revisores independentes utilizando os descritores saúde do homem, atenção primária a saúde e políticas de saúde nas bases de dados eletrônicas Scientific Electronic Library Online (SciELO), Literatura Científica e Técnica da América Latina e Caribe (LILACS) e Base de Dados em Enfermagem (BDENF).

Refinou-se, contemplando a terceira fase da pesquisa com a aplicação dos critérios de inclusão previamente estabelecidos na estratégia de busca: artigos publicados 


\section{artigo}

Oliveira, V.B.; Aguiar, R.S.

Conhecimento da política de saúde do homem e a relação com a atenção à saúde

de forma online nos últimos 6 anos (2014 a 2019); disponíveis em língua portuguesa; na íntegra; e no formato original oriundos de produções científicas diversificadas.

Leram-se criticamente, na quarta fase, os resumos dos estudos recuperados, excluindo os duplicados e aqueles cujo objetivo, resultados ou conclusão não mencionavam sobre a PNAISH.

Elaborou-se, para facilitar a avaliação e a análise dos dados, um instrumento que pudesse fornecer informações detalhadas dos estudos (Quadro 1). A extração dos dados foi conduzida por dois revisores independentes, sendo que os desacordos entre os revisores em relação aos dados extraídos eram discutidos, tendo como referência a publicação original. Extraíram-se variáveis de identificação tais como: periódico; país e ano de publicação; autor(es); título; delineamento; principais resultados e nível de evidência. Intentou-se com o instrumento, além de formar um banco de dados, mapear pontos pertinentes, integrar dados e caracterizar a amostra revisada.

Apresenta-se a seguir na figura 1 o fluxograma descritor dos resultados obtidos a partir da estratégia de busca de acordo com o fluxograma PRISMA?.

Resultou-se a busca na literatura um total
Figura 1 - Fluxograma da seleção dos estudos. Brasília, Distrito Federal, Brasil, 2019.

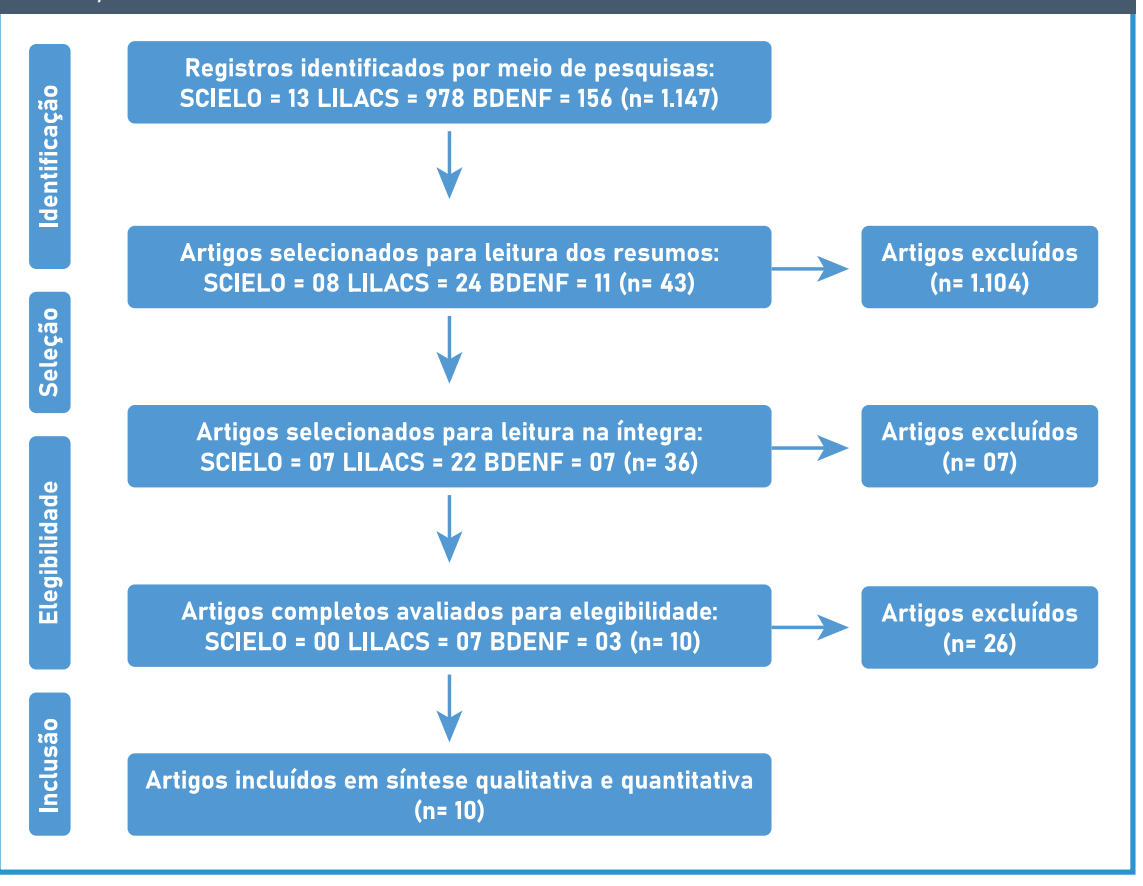

de 1.147 artigos capturados e, desses, 13 estavam no SCIELO, 978 na LILACS e 156 artigos na BDENF. Reduziu-se, a partir da aplicação dos filtros de inclusão, o número de ocorrência: SCIELO, recuperaram-se 08 (61,5\%) estudos; LILACS, 24 (2,45\%); e BDENF, 11 (7,05\%) estudos. Totalizaram-se 43 artigos submetidos à leitura dos resumos e à aplicação dos critérios de exclusão, gerando-se a rejeição de 1.104 artigos. Após a leitura completa dos artigos foram rejeitados ainda 33 artigos por não responderam à questão de pesquisa. Constituiu-se assim a amostra revisada de 10 artigos.

Classificaram-se as evidências dos artigos em seis níveis: Nível I - estudos relacionados à metanálise de múltiplos estudos controlados; Nível II - estudos experimentais individuais; Nível III - estudos quase-experimentais, como o ensaio clínico não randomizado, o grupo único pré e pós-teste, além de séries temporais ou caso-controle; Nível IV - estudos não experimentais, como a pesquisa descritiva, correlacional e comparativa, com abordagem qualitativa e estudos de caso; Nível V - dados de avaliação de programas obtidos de forma sistemática; e Nível VI - opinióes de especialistas, relatos de experiência, consensos, regulamentos e legislações ${ }^{10}$.

\section{RESULTADOS}

Apresenta-se no Quadro 1 a seguir as informações sobre os 10 artigos contidos nesta revisão integrativa. Foram interpretados e sintetizados todos os resultados, através de uma comparação dos dados evidenciados na análise dos artigos.

Quadro 1 - Distribuição dos artigos de acordo com o periódico, país, ano de publicação, autor(es), título, delineamento, resultados e nível de evidência. Brasilia, Distrito Federal, Brasil, 2019.

\begin{tabular}{|c|c|c|c|c|c|c|}
\hline & $\begin{array}{l}\text { PERIÓDICO, } \\
\text { PAÍS E ANO DE } \\
\text { PUBLICAÇÃO }\end{array}$ & AUTOR(ES) & TÍTULO & DELINEAMENTO & RESULTADOS & $\begin{array}{l}\text { NIIVEL DE } \\
\text { EVIDÊNCIA }\end{array}$ \\
\hline 总 & $\begin{array}{l}\text { Revista Online } \\
\text { de Pesquisa } \\
\text { Cuidado é } \\
\text { Fundamental, } \\
\text { Brasil, } 2015\end{array}$ & $\begin{array}{l}\text { Adamy EK, Trin- } \\
\text { dade LL, Teixei- } \\
\text { ra DC, Brambilla } \\
\text { DK, Galli KB }\end{array}$ & $\begin{array}{l}\text { Política Nacional de } \\
\text { Atenção Integral a } \\
\text { Saúde do Homem: } \\
\text { visão dos gestores } \\
\text { do SUS }\end{array}$ & $\begin{array}{l}\text { Pesquisa quali- } \\
\text { tativa de caráter } \\
\text { exploratória e } \\
\text { descritiva. }\end{array}$ & $\begin{array}{l}\text { Apontam fragilidades no discurso } \\
\text { sobre o conhecimento PNAISH. Os } \\
\text { resultados revelam que } 11(73,3 \%) \\
\text { gestores conhecem a PNAISH, três } \\
(20 \%) \text { conhecem parcialmente e um } \\
(6,66 \%) \text { disse não ter conhecimento } \\
\text { sobre a mesma. }\end{array}$ & IV \\
\hline
\end{tabular}




\begin{tabular}{|c|c|c|c|c|c|c|}
\hline $\begin{array}{l}N \\
0 \\
\text { 兽 } \\
\frac{1}{4}\end{array}$ & $\begin{array}{l}\text { Revista de } \\
\text { Enfermagem } \\
\text { da UFPI, Brasil, } \\
2015\end{array}$ & $\begin{array}{l}\text { Sousa AP, Silva } \\
\text { PES, Dias RS, } \\
\text { Azevedo PR, } \\
\text { Silva LDC }\end{array}$ & $\begin{array}{l}\text { Avaliação da } \\
\text { Política Nacional de } \\
\text { Atenção Integral à } \\
\text { Saúde do Homem }\end{array}$ & $\begin{array}{l}\text { Estudo } \\
\text { exploratório- } \\
\text { descritivo. }\end{array}$ & $\begin{array}{l}\text { Os fatores sociais e culturais } \\
\text { relacionados a organização do } \\
\text { serviço dificultam o acesso do } \\
\text { homem ao serviço preventivo, } \\
\text { destacando-se questões de } \\
\text { gênero. As barreiras estão } \\
\text { relacionadas ao horário de } \\
\text { funcionamento das unidades, } \\
\text { número de profissionais } \\
\text { insuficientes, redução de ações } \\
\text { educativas e ausência de } \\
\text { capacitação dos profissionais de } \\
\text { saúde. }\end{array}$ & IV \\
\hline $\begin{array}{l}m \\
0 \\
\text { 量 } \\
\frac{7}{2}\end{array}$ & $\begin{array}{l}\text { Revista de En- } \\
\text { fermagem UFPE } \\
\text { Online, Brasil, } \\
2018\end{array}$ & $\begin{array}{l}\text { Barbosa YO, } \\
\text { Menezes LPL, } \\
\text { Santos JMJ, } \\
\text { Cunha JO, Me- } \\
\text { nezes AF, Araú- } \\
\text { jo DC, Albuquer- } \\
\text { que TIP, Santos } \\
\text { AD }\end{array}$ & $\begin{array}{l}\text { Acesso dos homens } \\
\text { aos serviços de } \\
\text { atenção primária a } \\
\text { saúde }\end{array}$ & $\begin{array}{l}\text { Estudo quantita- } \\
\text { tivo, exploratório } \\
\text { e transversal. }\end{array}$ & $\begin{array}{l}\text { Foi identificado que } 32,6 \% \text { dos ho- } \\
\text { mens visitam os serviços de APS } \\
\text { com regularidade. A demora para } \\
\text { ser atendido }(35,7 \%) \text { e a ausência } \\
\text { de doenças }(33,8 \%) \text { são os princi- } \\
\text { pais fatores impeditivos da acessi- } \\
\text { bilidade masculina aos serviços de } \\
\text { saúde; e } 21 \% \text { desconhecem a Polí- } \\
\text { tica Nacional de Atenção Integral a } \\
\text { Saúde do Homem. }\end{array}$ & IV \\
\hline 茛 & $\begin{array}{l}\text { Escola Anna } \\
\text { Nery Revista de } \\
\text { Enfermagem, } \\
\text { Brasil, } 2014\end{array}$ & $\begin{array}{l}\text { Cavalcanti JRD, } \\
\text { Ferreira JA, } \\
\text { Henriques AHB, } \\
\text { Morais GSN, } \\
\text { Trigueiro JVS, } \\
\text { Torquato IMB }\end{array}$ & $\begin{array}{l}\text { Assistência integral } \\
\text { a saúde do homem: } \\
\text { necessidades, obs- } \\
\text { táculos e estratégias } \\
\text { de enfrentamento }\end{array}$ & $\begin{array}{l}\text { Estudo descritivo } \\
\text { e exploratório, } \\
\text { com abordagem } \\
\text { qualitativa. }\end{array}$ & $\begin{array}{l}\text { A população masculina tem neces- } \\
\text { sidades de saúde a serem atendi- } \\
\text { das e referenciam como obstáculos, } \\
\text { a vergonha de se expor, a impaciên- } \\
\text { cia, a inexistência de tempo e a falta } \\
\text { de resolutividade das necessidades } \\
\text { de saúde. }\end{array}$ & IV \\
\hline 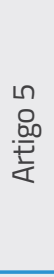 & $\begin{array}{l}\text { Escola Anna } \\
\text { Nery Revista de } \\
\text { Enfermagem } \\
\text { Brasil, } 2014\end{array}$ & $\begin{array}{l}\text { Pereira LP, Nery } \\
\text { AA }\end{array}$ & $\begin{array}{l}\text { Planejamento, ges- } \\
\text { tão e ações à saúde } \\
\text { do homem na es- } \\
\text { tratégia de saúde da } \\
\text { familia }\end{array}$ & $\begin{array}{l}\text { Estudo qualita- } \\
\text { tivo, descritivo e } \\
\text { exploratório. }\end{array}$ & $\begin{array}{l}\text { Não há atividades assistenciais } \\
\text { voltadas para os homens; não esti- } \\
\text { mulam a prevenção e a promoção à } \\
\text { saúde masculina; não desenvolvem } \\
\text { os processos de implantação da } \\
\text { atenção à saúde do homem, sendo } \\
\text { encontradas incipientes. }\end{array}$ & IV \\
\hline 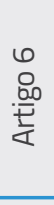 & $\begin{array}{l}\text { Escola Anna } \\
\text { Nery Revista de } \\
\text { Enfermagem, } \\
\text { Brasil, } 2014\end{array}$ & $\begin{array}{l}\text { Jesus MCP, San- } \\
\text { tos SMR, Lamas } \\
\text { JLT, Jesus PBR, } \\
\text { Gonçalves PLC, } \\
\text { Jory MV }\end{array}$ & $\begin{array}{l}\text { Marcadores de saú- } \\
\text { de do homem em } \\
\text { um município de pe- } \\
\text { queno porte }\end{array}$ & $\begin{array}{l}\text { Pesquisa descriti- } \\
\text { va, exploratória } \\
\text { com abordagem } \\
\text { quantitativa. }\end{array}$ & $\begin{array}{l}\text { As doenças cardiovasculares mos- } \\
\text { traram mais presentes entre os } \\
\text { homens, e sua alimentação inclui } \\
\text { um grande percentual de alimentos } \\
\text { processados. }\end{array}$ & IV \\
\hline 飡 & $\begin{array}{l}\text { Escola Anna } \\
\text { Nery Revista de } \\
\text { Enfermagem, } \\
\text { Brasil, } 2014\end{array}$ & $\begin{array}{l}\text { Araújo MG, } \\
\text { Lima GAF, Ho- } \\
\text { landa CSM, Car- } \\
\text { valho JBL, Sales } \\
\text { LKO }\end{array}$ & $\begin{array}{l}\text { Opinião de profis- } \\
\text { sionais sobre a efe- } \\
\text { tivação da Politica } \\
\text { Nacional de Atenção } \\
\text { Integral à Saúde do } \\
\text { Homem }\end{array}$ & $\begin{array}{l}\text { Pesquisa explo- } \\
\text { ratória/descritiva } \\
\text { com abordagem } \\
\text { qualitativa. }\end{array}$ & $\begin{array}{l}\text { Foi constatado que a PNAISH é } \\
\text { relevante para trabalhar as ações } \\
\text { para o homem. No entanto, o seu } \\
\text { processo formativo foi centrado na } \\
\text { atenção às doenças, fragmentado } \\
\text { e excessivamente biomédico, pro- } \\
\text { blematizando o desenvolvimento } \\
\text { de práticas de promoção da saúde. }\end{array}$ & IV \\
\hline
\end{tabular}




\section{artigo}

Oliveira, V.B.; Aguiar, R.S

Conhecimento da política de saúde do homem e a relação com a atenção à saúde

\begin{tabular}{|c|c|c|c|c|c|c|}
\hline 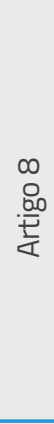 & $\begin{array}{l}\text { Revista Brasilei- } \\
\text { ra em Promoção } \\
\text { da Saúde, Brasil, } \\
2016\end{array}$ & $\begin{array}{ll}\text { Carneiro } & \text { LMR, } \\
\text { Santos } & \text { MPA, } \\
\text { Macena } & \text { RHM, } \\
\text { Vasconcelos TB }\end{array}$ & $\begin{array}{l}\text { Atenção Integral a } \\
\text { Saúde do Homem: } \\
\text { Um desafio na aten- } \\
\text { ção básica }\end{array}$ & $\begin{array}{l}\text { Estudo qualitati- } \\
\text { vo e descritivo. }\end{array}$ & $\begin{array}{l}\text { Ficou evidenciado que os profissio- } \\
\text { nais entrevistados não conhecem } \\
\text { a PNAISH e poucos compreendem } \\
\text { a sua importância para o acompa- } \\
\text { nhamento integral do homem. Há } \\
\text { várias dificuldades para a imple- } \\
\text { mentação da Política, tais como a } \\
\text { deficiência de recursos materiais e } \\
\text { humanos e ainda a falta de interes- } \\
\text { se do próprio homem em cuidar da } \\
\text { sua saúde. }\end{array}$ & IV \\
\hline $\begin{array}{l}0 \\
0 \\
.00 \\
\frac{1}{2}\end{array}$ & $\begin{array}{l}\text { Arquivos de } \\
\text { Ciências da Saú- } \\
\text { de da UNIPAR, } \\
\text { Brasil, } 2018\end{array}$ & $\begin{array}{l}\text { Assis NO, Ro- } \\
\text { drigues J, Chris- } \\
\text { tóforo BEB, Ta- } \\
\text { csi YRC }\end{array}$ & $\begin{array}{l}\text { Atuação dos en- } \\
\text { fermeiros frente à } \\
\text { Política Nacional de } \\
\text { Atenção Integral à } \\
\text { Saúde do Homem: } \\
\text { um estudo explora- } \\
\text { tório }\end{array}$ & $\begin{array}{l}\text { Pesquisa descriti- } \\
\text { va exploratória } \\
\text { com abordagem } \\
\text { qualitativa. }\end{array}$ & $\begin{array}{l}\text { A análise do modo como os en- } \\
\text { fermeiros desenvolvem a PNAISH } \\
\text { permitiu afirmar que ainda há ne- } \\
\text { cessidade de investir em capacita- } \\
\text { ção para os profissionais de enfer- } \\
\text { magem que trabalham nas UBS, } \\
\text { possibilitando assim a realização } \\
\text { de ações voltadas para população } \\
\text { masculina. }\end{array}$ & IV \\
\hline $\begin{array}{l}\text { 음 } \\
0 \\
.000 \\
\frac{10}{2}\end{array}$ & $\begin{array}{l}\text { Revista Baiana } \\
\text { de Saúde Públi- } \\
\text { ca, Brasil, } 2014\end{array}$ & $\begin{array}{l}\text { Nascimento LV, } \\
\text { Machado WD, } \\
\text { Gomes DF,Vas- } \\
\text { concelos MIO }\end{array}$ & $\begin{array}{l}\text { Estudo de avalia- } \\
\text { bilidade da Política } \\
\text { Nacional de Atenção } \\
\text { Integral à Saúde do } \\
\text { Homem no municí- } \\
\text { pio de Sobral, Ceará }\end{array}$ & $\begin{array}{l}\text { Pesquisa } \\
\text { exploratória } \\
\text { com abordagem } \\
\text { qualitativa. }\end{array}$ & $\begin{array}{l}\text { Os resultados revelam que a } \\
\text { PNAISH no município se encontra } \\
\text { estruturada, entretanto, existem } \\
\text { problemas relacionados à acessi- } \\
\text { bilidade, capacitação e qualificação } \\
\text { profissional, mobilização familiar } \\
\text { no incentivo à atenção à saúde dos } \\
\text { homens e principalmente mudança } \\
\text { física. }\end{array}$ & IV \\
\hline
\end{tabular}

No que tange ao ano de publicação, foi constatado que a maior quantidade foi publicada no ano de 2014 com cinco artigos (50\%), seguido de 2018 com dois (20\%), 2015 com dois (20\%) e 2016 com um artigo (10\%). O periódico de maior publicação foi a Escola Anna Nery Revista de Enfermagem com um total de quatro artigos (40\%). Quanto a profissão do primeiro autor dos artigos, em três $(30 \%)$ a primeira autoria era de enfermeira(o); em um (10\%) era de graduando em enfermagem; e em seis (60\%) não foram encontradas informações específicas relacionadas à sua formação, pois apresentava-se somente a vinculação acadêmica.

Quanto a região do país em que as pesquisas foram realizadas: sete (70\%) foram desenvolvidas na região Nordeste, uma (10\%) na região Sudeste, uma (10\%) na região Centro-Oeste e uma (10\%) na região Sul. Sobre os métodos utilizados, foi constatado que em oito artigos (80\%) foram utilizados o método qualitativo e em dois $(20 \%)$ o quantitativo.

\section{DISCUSSÃO}

Emergiram-se as seguintes categorias para discussão por meio de uma síntese narrativa: 1) Conhecimento dos profissionais de saúde sobre a PNAISH; 2) Conhecimento dos gestores das APS sobre a PNAISH; e 3) Fatores que dificultam o acesso do homem ao serviço de saúde e sua relação com o conhecimento de profissionais de saúde e gestores.

\section{Conhecimento dos profissionais de saúde sobre a PNAISH}

Evidencia-se através dos artigos que o conhecimento dos enfermeiros sobre a PNAISH ainda é escasso e apesar de saberem acerca da existência da política, não possuem conhecimento suficiente sobre a mesma, pois alegam falta de capacitação para prestarem atendimento à população masculina e que há pouco incentivo para se trabalhar com esse público ${ }^{11-12}$.

Os profissionais relatam conhecer a PNAISH, mas com limitações sobre os seus objetivos, princípios e diretrizes que norteiam a mesma ${ }^{12}$. Nessa perspectiva, a formação curricular da graduação é abordada como um fator importante para o despreparo por parte dos enfermeiros na abordagem aos homens, pois privilegia apenas a saúde das crianças, das mulheres e dos idosos?

Em estudo realizado em um município do sudoeste goiano com enfermeiros da APS, identificou-se que $80 \%$ deles não cursaram uma disciplina específica na graduação que abordasse a saúde do homem; relataram ainda que a abordagem ocorreu de forma generalista, superficial, fragmentada e descontextualizada do ambiente sociocultural dos homens em disciplinas 
como saúde coletiva, saúde do trabalhador e saúde do idoso ${ }^{11}$.

Dessa forma, destaca-se a importância de as escolas de enfermagem reavaliarem constantemente suas grades curriculares com objetivo de mantê-las atualizadas para instrumentalização dos graduandos de Enfermagem de modo a promoverem um atendimento de qualidade e de forma integral quando formados aos homens, pois na prática do dia-a-dia os profissionais acabam realizando um atendimento simples quando comparado ao da mulher e com um conhecimento pouco aprofundado, mesmo a política tendo diretrizes bem definidas e objetivos específicos para atender o público masculino na íntegra ${ }^{11}$.

Contudo, o estudo ${ }^{7}$ também destacou a necessidade de qualificação e capacitação permanente dos profissionais para promoverem a PNAISH, pois o enfermeiro capacitado tem a possibilidade de elaborar estratégias para que o homem esteja mais presente nos serviços. Assim, estudos evidenciam que o profissional de saúde deve buscar o conhecimento científico e se responsabilizar pela sua educação permanente para que eles estejam preparados para lidar com mitos, preconceitos e concepções errôneas que foram construídas no imaginário e no contexto social dos usuários ${ }^{13}$.

Enfatiza-se a necessidade do diálogo entre profissionais e gestores de saúde para a promoção eficiente da oferta de atenção ao homem e para que seja identificada a necessidade de capacitação de modo a promover a compreensão da PNAISH para que as ações de saúde direcionadas a este público possa ser realizada de modo eficaz e com a ajuda de todos ${ }^{14}$.

Nessa perspectiva, em estudo realizado No interior do Rio Grande do Norte (RN), um depoente remete a responsabilidade para a gestão municipal propondo espaços de capacitação com o intuito de favorecer o desenvolvimento de ações e práticas para o atendimento ao segmento masculino, pois os profissionais de saúde ainda não detêm o conhecimento para trabalhar com práticas voltadas para o homem, sendo necessária a inserção desses sujeitos em cursos de educação permanen- te de modo a identificar as necessidades de formação e o de-senvolvimento dos trabalhadores da área da saúde, além de construir estratégias e processos que qualifiquem a atenção e a gestão em saúde ${ }^{13}$.

\section{A função do gestor frente à PNAISH \\ é implantar}

mecanismos de

regulação das

atividades relativas à

política; coordenar,

acompanhar e avaliar

a PNAISH; e ainda

qualificar as equipes

de saúde para a

execução das ações

\section{propostas}

No estudo 2, realizado em três unidades de saúde de São Luís (MA), a grande maioria dos profissionais $(80 \%)$ referiram não terem treinamento direcionado ao acolhimento da população masculina, sendo que os mesmos tendem a destinar menos do seu tempo à saúde do homem devido a desvalorização de suas queixas. Diante disso, negligenciam o cuidado com a saúde do homem e acabam reforçando que a unidade é um ambiente feminino, fato que dificulta o acesso do público masculino aos serviços de saúde ${ }^{15}$.

O estudo 7 relata que as ações desenvolvidas pelos profissionais da Estratégia Saúde da Família (ESF) priorizam consultas individuais; valorizam a assistência médica; as consultas são rápidas; e os profissionais estão mais preocu-pados em oferecer uma pronta resposta, tomando decisões voltadas a condutas já conhecidas e centradas na terapêutica de patologias. $\mathrm{O}$ estudo salienta ainda que os profissionais de saúde, em sua maioria, consideram a PNAISH como um subsídio relevante para trabalhar ações voltadas para o homem, contudo, eles não possuem suporte suficiente para concretizar suas ações e, também, não conhecem a PNAISH em sua totalidade ${ }^{13}$.

Portanto, existe fragilidades para fornecer as informações aos profissionais de saúde em relação à PNAISH, colaborando assim para a falta de sensibilização dos profissionais em buscar informações para o aperfeiçoamento e desenvolvimento de ações direcionadas ao homem. Sendo assim, não são realizadas ações que contemplem especificamente a coletividade masculina ${ }^{12}$.

\section{Conhecimento dos gestores das APS sobre a PNAISH}

A função do gestor frente à PNAISH é implantar mecanismos de regulação das atividades relativas à política; coordenar, acompanhar e avaliar a PNAISH; e ainda qualificar as equipes de saúde para a execução das ações propostas ${ }^{16}$.

Diante disso, em estudo realizado em Santa Catarina (SC) onde foi questionado aos gestores sobre o seu papel na implantação e desenvolvimento da PNAISH, identificou-se que de forma geral os gestores não possuem clareza quanto as suas responsabilidades e competências necessárias para auxiliarem na implantação dessa política, sendo identificado ainda que eles direcionam somente às ações em busca de 
prevenção e nenhum deles resgatou aspectos relacionados à providência de recursos financeiros, estruturais, qualificação das equipes, formulação de estratégias de promoção da saúde, entre outros aspectos ${ }^{16}$.

O estudo 5 ressalta que a adesão ao Programa Nacional de Melhoria do Acesso e da Qualidade (PMAQ) representa um importante avanço para a promoção da saúde da população masculina, pois o programa fomenta o desenvolvimento de processos capazes de ampliar a capacidade da gestão - federal, estadual e municipal - na oferta de serviços que assegurem a melhoria do acesso e da qualidade da atenção à saúde de acordo com as necessidades concretas da população e que além desses aspectos, tem como um de seus desafios dirimir a incipiência dos processos de gestão ${ }^{14}$.

Porém, persistem as dificuldades relacionadas ao planejamento, gestão e execução da política devido a problemas orçamentários e financeiros que segundo os gestores é considerado como uma grande dificuldade para o planejamento e execução das ações nos serviços de saúde. Esse aspecto inclusive foi pontuado pelos gestores como uma justificativa para a tímida ou não implantação e desenvolvimento da política ${ }^{14,16}$.

Assim, é responsabilidade do gestor local buscar parcerias junto às demais esferas de governo para qualificar as equipes de saúde para a execução das ações propostas pela PNAISH, pois isso pode desenvolver novas competências e habilidades a fim de modificar a abordagem a esta população, proporcionando uma atuação mais qualificada e segura ${ }^{11}$.

Desse modo, torna-se necessário organizar e fortalecer o nível de gestão e o planejamento, reconhecendo a necessidade e $o$ interesse em desenvolver a atenção integral à saúde do homem na ESF ${ }^{14}$. Ademais, o gestor tem a obrigação de fiscalizar se os princípios do Sistema Único de Saúde (SUS) estão sendo respeitados, sendo de extrema importância que o mesmo discuta a saúde do homem e a inclua no Plano Municipal de Saúde e também na Programação Pactuada Integrada (PPI) para que possa estabelecer metas e dispor de recursos financeiros para ações e capacita- ções da equipe, bem como definir prazos e indicadores que desejam ser alcançados ${ }^{16}$.

O estudo 9 enfatiza que é importante criar momentos de discussão entre os profissionais, juntamente com a gestão, para assim discutir sobre o estabelecimento da educação continuada para a efetivação do serviço de saúde, pois o conhecimento de ambos sobre a política ainda é escasso o que impossibilita o desenvolvimento de ações eficazes para acolher os homens nos serviços de saúde ${ }^{11}$.

Diante disso, a gestão pode ser parceira na realização de ações possibili-tando o acesso do segmento masculino nos espaços de saúde, subsidiando uma atenção voltada para a integralidade das ações e dos sujeitos envolvidos, entendendo suas singularidades e necessidades em saúde, podendo identificar oportunidades de aproximação com o homem extrapolando os espaços físicos da unidade básica de saúde $(\text { UBS })^{13}$.

Portanto, ressalta-se que existe nacionalmente uma dificuldade dos gestores na elaboração de mecanismos para a aproximação e participação dos homens nos serviços de saúde, sendo que a falta de conhecimento acerca da PNAISH pode interferir nas ações de planejamento. Dessa forma, a PNAISH se encontra em estado de incipiência visto que suas estratégias não são realizadas ${ }^{14}$.

\section{Fatores que dificultam o acesso do homem ao serviço de saúde e sua relação com o conhecimento de pro- fissionais de saúde e gestores}

Identifica-se nos estudos que os homens não visitam regularmente os serviços de APS, não realizam exames laboratoriais com frequência, desconhecem a PNAISH e referem que os profissionais de saúde não estão capacitados o suficiente para prestar uma boa assistência a eles ${ }^{12,17-18}$.

$\mathrm{Na}$ literatura, já está bem evidenciado que existem várias causas que impedem os homens de frequentar as UBS. Nesse sentido, os artigos 3 e 4 abordam os fatores que dificultam a acessibilidade nos serviços de saúde17-18, sendo a demora em ser atendido, a ausência de doenças, o medo de descobrir doença grave e a falta de acolhimento por parte dos profissionais de saúde os mais comuns ${ }^{17}$.

Constata-se que outro fator que interfere na adesão masculina aos serviços de saúde é a vinculação que eles fazem em relação a realização obrigatória do exame preventivo do câncer de próstata e ainda pela falta de resolutividade na assistência ${ }^{18}$.

Além de só procurarem algum serviço de saúde quando já acometido por alguma enfermidade ou por problemas agudos, o estudo 8 afirma que a masculinidade construída sócio culturalmente ainda prevalece, trazendo a ideia de que o homem nunca adoece ou que é um ser invulnerável. Porém, é importante ressaltar que não só a questão cultural dificulta a adesão do homem aos serviços de saúde, mas as questões de acesso ao serviço também constituem uma barreira ${ }^{12}$. Além disso, outras situações que também podem levar os homens a não procurarem atendimento na APS são: falta de tempo, incompatibilidade dos horários, impaciência, vergonha de se expor, falta de especialistas e de resolutividade das necessidades de saúde e a ideia de que a UBS é um espaço somente para a mulher ${ }^{17}$.

Diante disso, reconhece-se que de fato há predominância feminina nas atividades da APS, sendo elas a maioria nas consultas, nas salas de espera, nas filas, nos grupos e nas áreas de circulação das UBS. Comprova-se, assim, que os homens associam os cuidados com a saúde ao universo feminino ao considerar as mulheres como seres mais frágeis e mais vulneráveis às doenças e que esse cuidado é um papel somente das mulheres ${ }^{17 .}$

Nessa perspectiva, os estudos 1 e 10 mostraram que isso se deve a existência de vários programas de saúde específicos para o sexo feminino e a escassez de açôes específicas para indivíduos do sexo masculino nos diversos níveis de atenção, favorecendo a dificuldade de interação entre o público masculino e os serviços de saúde $^{16,19}$. No estudo 2, os autores relatam que algumas UBS não realizam nenhuma ação para a saúde do homem e que existe, portanto, a necessidade de programas vol- 
tados para a população masculina já que eles possuem demandas específicas, como qualquer outro grupo ${ }^{15}$.

Em outro estudo onde se abordou a temática do acesso do homem ao serviço de saúde, os autores destacaram alguns impedimentos que levam a resistência dos homens a UBS, dentre eles estão os obstáculos organizacionais do SUS, como as dificuldades relacionadas ao espaço físico insuficiente; a falta de recursos materiais nas unidades para o desenvolvimento das ações básicas de atenção à saúde; a falta de recursos humanos para atender a demanda espontânea da população masculina; o enfrentamento de filas intermináveis que, muitas vezes, causam a perda de um dia inteiro de trabalho, sem que necessariamente tenham suas demandas resolvidas em uma única consulta; a ausência de acolhimento pela falta de capacitação dos profissionais com a saúde masculina; o horário de funcionamento das unidades de saúde que é incompatível com a longa jornada de trabalho da população masculina, e que dificilmente encontram-se abertos após as 17 horas, restando aos homens apenas os serviços de emergência/ urgência que possuem atendimento nas 24 horas, superlotando-os muitas vezes com demandas que poderiam ser sanadas na APS15. Diante disso, o estudo 5 relata que nos serviços em que houve uma disponibilização de atendimento aos sábados e domingos, terceiro turno (noturno) ou até mesmo atendimento 24 horas, houve uma maior presença masculina do que naqueles que não flexibilizaram os horários ${ }^{14}$.

Constata-se, dessa forma, que a procura dos serviços de saúde pelos homens é devido a presença de alguma doença, pela busca de medicamentos ou como acompanhante dos filhos ou da esposa, ignorando as suas consultas de caráter preventivo ${ }^{20}$.

O estudo 8 destaca algumas açôes direcionadas ao homem que são realizadas nas UBS, como a campanha do Novembro Azul - específica ao público masculino - que trabalha a prevenção do câncer de próstata, incentivando-os a fazer o exame de PSA e de toque retal ${ }^{12}$, porém existem falhas na campanha, começando pela fra- gilidade do conhecimento dos profissionais sobre a temática, como a falta de informações sobre os fatores de risco, sobre quem de fato deve fazer o exame, sobre a idade recomendada para o rastreamento, sobre a sintomatologia da doença e, caso tenha necessidade de se fazer o exame, como ele é realizado ${ }^{13}$. Dessa forma, a campanha acaba causando um obstáculo entre os homens e os serviços de saúde, pois eles não aderem, muitas vezes, às medidas de prevenção e promoção da saúde pela vergonha de se expor devido ao modelo hegemônico da masculinidade, pois o exame está associado à violação do ser masculino ${ }^{18}$.

Um estudo realizado no estado de Sergipe (SE) sobre a proximidade da população masculina com a equipe de saúde constatou que poucos participantes conheciam o agente comunitário de saúde (ACS), o enfermeiro e o médico responsáveis pela área de abrangência na qual os mesmos residiam. Com isso, entende-se que esta não vinculação dos homens com a equipe de saúde da APS representa a deficiência dos serviços no desenvolvimento de ações voltadas à saúde do homem distanciando, assim, o alcance dos objetivos da PNAISH ${ }^{17}$.

Outro fator que distancia o homem dos serviços de saúde é a demora do atendimento, visto que o número insuficiente de profissionais ou a falta de organização nos atendimentos acaba superlotando a unidade e dificultando a agilidade que o homem procura, pois na maioria das vezes em que eles se encontram na UBS estão ausentes de suas atividades laborais, além da falta de um atendimento acolhedor, com uma comunicação ineficaz, prejudicando as relações profissionais e usuários e dificultando a adesão dos homens ao serviço ${ }^{18}$.

Para que o homem tenha uma inclusão nos serviços de saúde, é preciso que sua política seja trabalhada de forma integral, com um processo dinâmico e complexo, por meio de uma reorganização das ações de saúde através de uma proposta inclusiva. Diante disso, o estudo 8 ressalta que não há dificuldades para implementar a PNAISH na UBS, porém deve-se ter o empenho de toda a equipe para se capacitarem, desde o enfermeiro que presta a assistência até os gestores que administram, com isso as ações de promoção da saúde e prevenção de doenças poderiam ser intensificadas através de campanhas de conscientização e educação, sobre diversas temáticas que chamassem a atenção do homem ${ }^{12}$.

Assim, existe uma necessidade crucial de promover capacitações dos gestores e dos profissionais atuantes na APS, de modo a promover a compreensão da PNAISH, bem como o conhecimento das nuances do trabalho de atenção à saúde da população masculina, visto que, segundo a PNAISH a capacitação técnica dos profissionais de saúde para o atendimento do homem é imprescindível para que os princípios da humanização e da qualidade que implicam na promoção, no reconhecimento dos direitos dos homens e no respeito à ética sejam cumpridos ${ }^{14,21-23}$.

\section{CONCLUSÃO}

Os profissionais da APS têm conhecimento escasso acerca da PNAISH e os poucos que sabem da sua existência, conhecem com limitações, pois alegam que há pouco incentivo para se trabalhar com o público masculino por parte da gestão, já que na maioria das vezes realizam capacitações para outros públicos e raramente para a população masculina. Essa desvalorização à saúde do homem começa desde a graduação onde, na maioria dos cursos, não há disciplina específica para a saúde do homem. Ademais, os gestores não possuem clareza quanto as suas responsabilidades para auxiliarem na implantação da política, sendo que muitos deles não possuem conhecimento sobre a existência da mesma dificultando, assim, o incentivo que os profissionais precisam para se capacitarem.

Portanto, os homens não comparecem regulamente na APS, pois acabam encontrando dificuldades para sua adesão. Assim, o pouco conhecimento dos profissionais e gestores torna-se um fator prejudicial para a formação de vínculo e desenvolvimento de ações específicas para o público masculino nas UBS. - 


\section{REFERÊNCIAS}

1. Vasconcelos ICBL, Prestes JYN, Ribeiro RRS, Lima SJL, Farias SDCF, Barbosa LDS et al. Política nacional de atenção integral a saúde do homem e os desafios de sua implementação. Braz J of Develop [Internt]. 2019 [acesso em 2019 Nov 10];5(9):1634016355. DOI: $10.34117 /$ bjdv5n9-185

2. Pereira J, Klein C, Meyer DE. PNAISH: uma análise de sua dimensão educativa da perspectiva de gênero. Saúde Soc [Internet]. 2019 [acesso em 2019 Nov 10];28(2):132-146. DOl: $10.1590 / S 0104-12902019170836$

3. Carneiro VSM, Adjuto RNP, Alves KAP. Saúde do homem: identificação e análise dos fatores relacionados à procura ou não, dos serviços de atenção primária. Arq Ciências Saúde UNIPAR [internet]. 2019 [acesso em 2019 Nov 10];23(1):35-40. DOI: 10.25110/arqsaude.v23i1.2019.6521

4. Cesaro BC, Santos HB, Silva FNM. Masculinidades inerentes à política brasileira de saúde do homem. Rev Panam Salud Pública [Internet]. 2018 [acesso em 2019 Nov 10];42:e119. DOI: https:// doi.org/10.26633/RPSP.2018.119

5. Miranda SVC, Oliveira PSD, Moraes VCM, Vasconcelos LCF. Necessidades e reivindicações de homens trabalhadores rurais frente à atenção primária à saúde. Trab Educ Saúde [Internet]. 2019 [acesso em 2019 Nov 10]18(1):e0022858. DOl: 10.1590/1981-7746-sol00228

6. Silva AN, Silva AS, Silva ARV, Araújo TME, Rebolças CBA, Nogueira LT. A avaliação da atenção primária a saúde na perspectiva da população masculina. Rev Bras Enferm [Internet]. 2018 [acesso em 2019 Nov 10];71(2):255-63. DOI: 10.1590/00347167-2016-0651

7. Aguiar RS, Santana DC, Santana PC. A percepção do enfermeiro da estratégia saúde da família sobre a saúde do homem. Rev Enferm Cent-Oeste Min [Internet]. 2015 [acesso em 2019 Jun 05];5(3):1844-54. DOI: 10.19175/recom.v5i3.872

8. Donato $\mathrm{H}$, Donato M. Etapas na condução de uma revisão sistemática. Acta Med Port [Internet]. 2019 [acesso em 2019 Set 22];32(3):227-235. DOI: 10.20344/amp.11923

9. Moher D, Liberati A, Tetzlaff J, Altman DG, The PRISMA Group. Preferred reporting items for systematic reviews and meta-analyses: the PRISMA statement. PLoS Med [Internet]. 2009 [acesso em 2019 Set 24];6(7):e1000097. DOl: 10.1371/journal. pmed.1000097

10. OCEBM Levels of Evidence Working Group. The Oxford 2011 Levels of Evidence [Internet]. Oxford: Oxford Centre for Evidence-Based Medicine; 2011. Disponivel em: https://www.cebm. net/2016/05/ocebm-levels-of- evidence/

11. Assis NO, Rodrigues J, Christóforo BEB, Tacsi YRC. Atuação dos enfermeiros frente à Política Nacional de Atenção Integral à Saúde do Homem: um estudo exploratório. Arq Ciências Saúde UNIPAR [Internet]. 2018 [acesso em 2019 Set 13];29(4):554563. DOI: 10.25110/arqsaude.v22i3.2018.6397

12. Carneiro LMR, Santos MPA, Macena RHM, Vasconcelos TB. Atenção Integral a Saúde do Homem: um desafio na atenção básica. Rev Bras Promoç Saúde [Internet]. 2016 [acesso em 2019
Set 13];29(4):554-563. DOI: 10.5020/18061230.2016.p554

13. Araújo MG, Lima GAF, Holanda CSM, Carvalho JBL, Sales LKO. Opinião de profissionais sobre a efetivação da Politica Nacional de Atenção Integral à Saúde do Homem. Esc Anna Nery Rev Enferm [Internet]. 2014 [acesso em 2019 Set 13];18(4):682-689. DOI: 10.5935/1414-8145.20140097

14. Pereira LP, Nery AA. Planejamento, gestão e ações à saúde do homem na estratégia de saúde da família. Esc Anna Nery Rev Enferm [Internet]. 2014 [acesso em 2019 Set 13];18(4):635643. DOI: $10.5935 / 1414-8145.20140090$

15. Sousa AP, Silva PES, Dias RS, Azevedo PR, Silva LDC. Avaliação da Política Nacional de Atenção Integral à Saúde do Homem. Rev Enferm UFPI [Internet]. 2015 [acesso em 2019 Set 12];4(3):65-70. DOI: 10.26694/reufpi.v4i3.4093

16. Adamy EK, Trindade LL, Teixeira DC, Brambilla DK, Galli KB. Política Nacional de Atenção Integral a Saúde do Homem: visão dos gestores do SUS. Rev Pesqui (Univ Fed Estado Rio J Online) [Internet]. 2015 [acesso em 2019 Set 12];7(2):2415-2424. DOI: 10.9789/2175-5361.2015.v7i2.2415-2424

17. Barbosa YO, Menezes LPL, Santos JMJ, Cunha JO, Menezes AF, Araújo DC et al. Acesso dos homens aos serviços de atenção primária a saúde. Rev Enferm UFPE On line [Internet]. 2018 [acesso em 2019 Set 12];12(11):2897-905. doi: 10.5205/1981-8963-v12i11a237446p2897-2905-2018

18. Cavalcanti JRD, Ferreira JA, Henriques AHB, Morais GSN, Trigueiro JVS, Torquato IMB. Assistência integral a saúde do homem: necessidades, obstáculos e estratégias de enfrentamento. Esc Anna Nery Rev Enferm [Internet]. 2014 [acesso em 2019 Set 13];18(4):628-634. DOI: 10.5935/1414-8145.20140089

19. Nascimento LV, Machado WD, Gomes DF, Vasconcelos MIO. Estudo de avaliabilidade da Política Nacional de Atenção Integral à Saúde do Homem no município de Sobral, Ceará. Rev Baiana Saúde Pública [Internet]. 2014 [acesso em 2019 Set 13];38(1),95-114. DOI: 10.5327/z0100-0233-2014380100015

20. Jesus MCP, Santos SMR, Lamas JLT, Jesus PBR, Gonçalves PLC, Jory MV. Marcadores de saúde do homem em um município de pequeno porte. Esc Anna Nery Rev Enferm [Internet]. 2014 [acesso em 2019 Set 13];18(4):650-655. DOI: 10.5935/14148145.20140092

21. Balica LO, Aguiar RS. Percepções paternas no acompanhamento do pré-natal. Rev aten saúde [Internet]. 2019 [acesso em 2020 Jan 13];17(61):114-126. DOI: 10.13037/ras. vol117n61.5934

22. Lima CS, Aguiar RS. Acesso dos homens aos serviços de atenção primária à saúde: uma revisão integrativa. Research, Society and Development [Internet]. 2020 [acesso em 2020 Fev 13];9(4):e157943027. DOI: 10.33448/rsd-v9i4.3027

23. Lima RC, Aguiar RS. Experiência paterna com o recém-nascido a partir das orientações de enfermagem. Rev Cereus [Internet]. 2020 [acesso em 2020 Fev 13];12(1):193-202. DOI: 10.18605/2175-7275/cereus.v12n1p193-202 